\title{
Partícula na Caixa
}

\section{- um programa didáctico do modelo da partícula em poço de potencial para microcomputadores da família Apple II}

Alexandre L. Magalhães ${ }^{a}$

J. A. N. F. Gomes ${ }^{a}$

\section{Introdução}

A resolução analítica exacta da equação de Schrödinger só é possível para sistemas materiais tão simples como o átomo de hidrogénio ou iões hidrogenóides. A obtenção de energias e funções de estado de sistemas atómicos e moleculares mais complexos terá de ser feita por intermédio de métodos numéricos de aproximação, como o perturbacional e o variacional, que conduzem a soluções não exactas.

Existem, no entanto, certos modelos que permitem a resolução analítica da equação de Schrödinger e que se revelam bastante úteis na compreensão de certos fenómenos de natureza química. Um desses modelos é o da partícula numa caixa ou poço de potencial.

O programa PARTÍCULA NA CAIXA constitui um apoio didáctico à apresentação teórica do modelo da partícula em poço de potencial, permitindo o controlo de parâmetros como o número quântico, comprimento da caixa e valor do potencial de uma das paredes do poço, assim como a rápida visualização dos seus efeitos nas soluções analíticas fornecidas por este modelo. Este programa proporciona, deste modo, uma assimilação mais completa de certos conceitos quânticos importantes inerentes ao modelo, nomeadamente, a quantificação da energia, densidade de probabilidade de presença da partícula, pontos nodais e efeito túnel.

\section{Modelo da Partícula na Caixa}

No presente trabalho procuram-se as soluções de estacionaridade e, por isso, trabalhar-se-á com a equação de Schrödinger independente do tempo:

$\hat{\mathrm{H}} \Psi=\mathrm{E} \Psi \Leftrightarrow(\hat{\mathrm{E}} \mathrm{c}+\hat{\mathrm{E} p}) \Psi=\mathrm{E} \Psi$

Considere-se então o caso unidimensional (por exemplo segundo a direcção do eixo xx') de uma partícula de massa m sujeita a um potencial da seguinte forma:

$V(x)= \begin{cases}\infty & ; \mathrm{x}<0 \mathrm{~V} x>\mathrm{L} \\ 0 & ; 0<\mathrm{x}<\mathrm{L}\end{cases}$

Como o potencial é descontínuo, é conveniente escrever uma equação de Schrödinger para cada região de diferente potencial.
Para regiões de $\mathrm{V}(\mathrm{x})=\infty$ obtém-se:

$\left(-h^{2} / 2 \mathrm{~m}\right) \mathrm{d}^{2} \Psi_{1}(\mathrm{x}) / \mathrm{dx}^{2}+\infty \Psi_{1}(\mathrm{x})=\mathrm{E} \Psi_{1}(\mathrm{x}) ; \mathrm{x}<0 \mathrm{~V} \mathrm{x}>\mathrm{L}$

Para regiões de $\mathrm{V}(\mathrm{x})=0$ :

$\left(-\mathrm{h}^{2} / 2 \mathrm{~m}\right) \mathrm{d}^{2} \Psi_{\|}(\mathrm{x}) / \mathrm{dx}^{2}=\mathrm{E} \Psi_{\|}(\mathrm{x}) ; 0<\mathrm{x}<\mathrm{L}$

As soluções destas duas equações são respectivamente:

$\Psi_{1}(\mathrm{x})=0$

$\Psi_{\|}(\mathrm{x})=\mathrm{C} \cos (\mathrm{kx})+\mathrm{D} \sin (\mathrm{kx})$

onde $\mathrm{k}=\sqrt{2 \mathrm{mE}} / \mathrm{h}$ e C,D são constantes de integração.

Para garantir a continuidade da função a todo o domínio impõe-se que:

$\Psi_{\|}(0)=\Psi_{\|}(L)=0$

Daqui se conclui que a constante $\mathrm{C}$ e a quantidade $\sin (\mathrm{kL})$, presentes na expressão (5) terão de ser ambas nulas:

$\mathrm{C}=0$

$\mathrm{e}$

$\sin (\mathrm{kL})=0 \Rightarrow \mathrm{kL}=\mathrm{n} \pi ; \mathrm{n}=1,2,3 \ldots$

A função na região $0<\mathrm{x}<\mathrm{L}$ tomará então a seguinte forma:

$\Psi_{\|}(\mathrm{x})=\mathrm{D} \sin ((\sqrt{2 \mathrm{mE}} / \mathrm{h}) \mathrm{x})=\mathrm{D} \sin (\mathrm{n} \pi \mathrm{x} / \mathrm{L})$

A constante D será calculada pela condição de normalização, de acordo com a interpretação de Born para $\Psi$ :

$f_{D} \Psi^{*} \Psi d \tau=1 \Rightarrow \int_{-\infty}^{+\infty} \Psi^{*}(x) \Psi(x) d x=1$

Obtêm-se finalmente para este sistema as funções de estado:

\footnotetext{
a Grupo de Química Teórica e Biofísica

Departamento de Química

Faculdade de Ciências, Universidade do Porto

Praça Gomes Teixeira

4000 Porto
} 
$\Psi_{n}(x)=\left\{\begin{array}{cl}0 & ; x<0 V x>L \\ \sqrt{2 / L} \sin (n \pi x / L) & ; 0<x<L\end{array}\right.$

com as respectivas energias, deduzidas de (7):

$E_{n}=n^{2} h^{2} / 8 m L^{2}$

$\mathrm{O}$ número $\mathrm{n}$, que surge pela imposição de uma condição fronteira, pode tomar valores inteiros positivos e é designado por número quântico.

\section{Análise das soluções obtidas}

Sob o ponto de vista didáctico, os aspectos mais importantes a salientar das soluções apresentadas em (10) e (11) são os seguintes:

- Em resultado das condições fronteira impostas, as energias só podem tomar valores discretos. Diz-se então que a energia está quantificada.

- A dependência da energia em $\mathrm{n}^{2}$, faz com que o espaçamento entre níveis de energia aumente com o $\mathrm{n}^{\circ}$ quântico:

$E_{n+1}-E_{n}=(2 n+1) h^{2} / 8 m L^{2}$

- A dependência em $\mathrm{L}^{-2}$, mostra que os níveis de energia e o seu espaçamento tomam valores superiores, quando se força a partícula a uma região mais restricta.

- A energia mínima que a partícula pode possuir não é nula (energia do ponto zero), como é de esperar, de modo a que o Princípio da Incerteza de Heisenberg seja obedecido.

- O número de nodos (pontos onde a função se anula) aumenta com n.

- Uma característica das funções que importa ainda salientar é o facto de serem ortogonais entre si, obedecendo portanto à relação:

$f_{D} \Psi_{n} \Psi_{m} d \tau=\delta n m$

\section{Caixa de potencial unidimensional com uma das paredes finita}

Considere-se a caixa apresentada anteriormente, baixando agora o potencial de uma das paredes para um valor finito $\mathrm{V}_{\text {。 }}$. A partícula fica assim sujeita à seguinte função de potencial:

$$
\mathrm{V}(\mathrm{x})= \begin{cases}\infty & ; \mathrm{x}<0 \\ 0 ; & (\text { REGIÃO I) } \\ \mathrm{V}_{\mathrm{o}} ; \mathrm{x}>\mathrm{L} & \text { (REGIÃO II) }\end{cases}
$$

Para a REGIÃO I será nula.

Para a REGIÃO II as soluções são dadas pela resolução da equação de Schrödinger (4). Neste caso, porém, só se pode impor uma condição fronteira, obrigando a função a anular-se somente no ponto $\mathrm{x}=0$, pois em $\mathrm{x}=\mathrm{L}$ o potencial não é infinito. A solução para esta região será, então, dada por:

$\Psi_{\|}(\mathrm{x})=\mathrm{A}_{\|} \sin \left(2 \pi \mathrm{x} / \lambda_{\|}\right)$ onde $\lambda_{\|}=\mathrm{h} / \sqrt{2 \mathrm{mE}}$ e $\mathrm{A}_{\|}$é uma constante que se determinará no fim, pela condição de normalização da função total.

Para a REGIÃO III a equação de Schrödinger tomará a seguinte forma:

$\left(-h^{2} / 2 m\right) d^{2} \Psi(x) / d x^{2}+V_{o} \Psi(x)=E \Psi(x)$

e terá de se considerar dois casos distintos: $\mathrm{E}<\mathrm{V}_{\mathrm{o}} \mathrm{e} \mathrm{E}>\mathrm{V}_{\mathrm{o}}$. No primeiro caso, a partícula encontra-se num estado ligado, não possuindo energia suficiente para passar a barreira de potencial. A solução da equação (16) é semelhante à apresentada em (5), preferindo-se aqui a seguinte forma alternativa:

$\Psi_{\| I}(\mathrm{x})=\mathrm{A}_{\| \mid} \exp \left(\mathrm{i} 2 \pi \mathrm{x} / \lambda_{\| 11}\right)+\mathrm{B}_{\| \mid} \exp \left(-\mathrm{i} 2 \pi \mathrm{x} / \lambda_{\| 1}\right)$

$\operatorname{com} \lambda_{\| 1}=h / \sqrt{2 m\left(E-V_{o}\right)}$. A forma (17) é mais conveniente, dado que $\lambda_{\| \mid}$é uma quantidade imaginária, e assim os argumentos das exponenciais serão reais.

Para que esta função seja aceitável para descrever um estado ligado, ela deve anular-se quando $\mathrm{x}$ tende para infinito. Isto implica que $B_{\|||}$seja nulo:

$$
\lim _{x \rightarrow+\infty} \Psi_{\mathrm{II}}(\mathrm{x})=0 \Rightarrow \Psi_{\mathrm{II}}(\mathrm{x})=\mathrm{A}_{\mathrm{II}} \exp \left(\mathrm{i} 2 \pi \mathrm{x} / \lambda_{\mathrm{II}}\right)
$$

Uma função de estado aceitável deve ainda ser unívoca, contínua e de primeira derivada contínua (excepto nos pontos onde o potencial é infinito). Assim, devem impor-se condições ao unir os dois fragmentos (15) e (18) no ponto $\mathrm{x}=\mathrm{L}$ :

Para garantir a continuidade da função:

$\mathrm{A}_{\|} \sin \left(2 \pi \mathrm{L} / \lambda_{\|}\right)=\mathrm{A}_{\|} \exp \left(+\mathrm{i} 2 \pi \mathrm{L} / \lambda_{\|\|}\right)$

Para garantir a continuidade na $1^{\underline{a}}$ derivada:

$\left(2 \pi / \lambda_{\|}\right) \mathrm{A}_{\|} \cos \left(2 \pi \mathrm{L} / \lambda_{\|}\right)=\left(\mathrm{i} 2 \pi / \lambda_{\|||}\right) \mathrm{A}_{\| \mid} \exp \left(+\mathrm{i} 2 \pi \mathrm{L} / \lambda_{\|\|}\right)$

Estas duas equações podem ser rearranjadas numa só, conduzindo à expressão:

$\tan \left(2 \pi \mathrm{L} / \lambda_{\|}\right)=-\mathrm{i} \lambda_{\| \mid} / \lambda_{\|}$

Substituindo $\lambda_{\|}$e $\lambda_{\| \mid}$pelas correspondentes expressões, obtém-se a equação:

$\tan (2 \pi \mathrm{L} \sqrt{2 \mathrm{mE}} / \mathrm{h})=-\mathrm{E} / \sqrt{\mathrm{V}_{\mathrm{o}}-\mathrm{E}}$

A única incógnita presente nesta igualdade é a energia $\mathrm{E}$. Para um dado conjunto de valores de $\mathrm{L}, \mathrm{m} \mathrm{e}_{\mathrm{o}}$, só certas energias $\mathrm{E}<\mathrm{V}_{\mathrm{o}}$ satisfazem (22) podendo ser determinadas através de um processo gráfico. (No programa PARTíCULA NA CAIXA as resoluções desta equação foram efectuadas com o electrão $\left(\mathrm{m}=9.11 \times 10^{-31} \mathrm{Kg}\right)$ e um comprimento da caixa $\mathrm{L}=25 \AA$ ).

Encontrados os valores de E permitidos para a partícula, podem calcular-se $\lambda_{\|} \mathrm{e} \lambda_{\| !}$. Para o cálculo das constantes $\mathrm{A}_{\|} \mathrm{e}$ $\mathrm{A}_{\|}$, utiliza-se a condição de normalização da função total e a razão $A_{||} / A_{||}$obtida com a equação (19). 
No caso da energia $\mathrm{E}$ ser superior a $\mathrm{V}_{\mathrm{o}}$, o comprimento de onda $\lambda_{\|||}$é agora uma quantidade real. Prefere-se então para a solução geral da equação de Schrödinger referente à REGIÃO III, a seguinte forma:

$\Psi_{\|||}(\mathrm{x})=\mathrm{C}_{\|||} \sin \left(2 \pi \mathrm{x} / \lambda_{\|||}\right)+\mathrm{D}_{\|||} \cos \left(2 \pi \mathrm{x} / \lambda_{\|||}\right)$

A função total é uma sinusoide que oscila indefinidamente a partir do ponto $\mathrm{x}=0$, não se encontrando a partícula num estado ligado. Qualquer que seja $\mathrm{E}>\mathrm{V}_{\mathrm{o}}$, é sempre possível ajustar a função $\Psi_{\| \mid l}(\mathrm{x})$ à função $\Psi_{\| 1}(\mathrm{x})$ no ponto $\mathrm{x}=\mathrm{L}$ (escolhendo convenientemente $\mathrm{C}_{1 \mid}$ e $\mathrm{D}_{\text {ifl }}$ ). Isto tem como consequência a não quantificação dos valores de E, obtendo-se assim um espectro contínuo de energia.

\section{Programa PARTÍCULA NA CAIXA}

Com base neste modelo, foi elaborado um programa de muito fácil utilização, designado por PARTÍCULA NA CAIXA. Este trata o problema unidimensional da partícula de massa $\mathrm{m}$, sujeita a dois tipos diferentes de função de potencial: poço de potencial infinito e poço de potencial com uma das paredes finita.

Com a execução deste programa, podem controlar-se parâmetros como o número quântico, comprimento da caixa, e valor do potencial de uma das paredes do poço, visualizando-se rapidamente os efeitos, nas soluções analíticas fornecidas por este modelo. O programa PARTÍCULA NA CAIXA permite, deste modo, a familiarização com certos conceitos quânticos importantes subjacentes ao modelo, podendo constituir um útil apoio didáctico à respectiva apresentação teórica.

\section{Características do programa e recursos necessários à sua utilização}

O programa foi elaborado em linguagem APPLESOFT/ BASIC, e é apresentado em disco flexível de 5.25 polegadas. Para a sua utilização será necessário um microcomputador APPLE IIc, ou APPLE IIe com leitor de discos (DRIVE) apropriado.

Existem duas versões deste programa:

VERSÃO 1 - Apple Ile e Apple IIc - DOS 3.3 - APPLESOFT/BASIC não compilado.

VERSÃO 2 - Apple IIe e Apple IIc - ProDOS - APPLESOFT/BASIC compilado (compilação feita com o programa BEAGLE COMPILER).

O programa apesar de extenso, não carece de memória adicional, pelo que $64 \mathrm{~K}$ de memória RAM, característico do Apple Ile, é suficiente para a sua execução.

\section{Estrutura do programa}

Este programa geral é constituído por vários subprogramas, que se interligam como é mostrado no seguinte esquema:

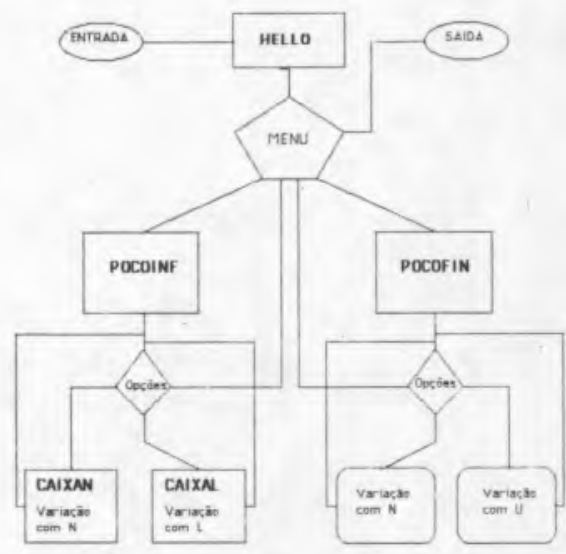

Os subprogramas que constituem o programa PARTÍCULA NA CAIXA têm as seguintes funções:

- Programa HELLO: Corre automaticamente quando o sistema é accionado, apresentando uma lista de opções que permite escolher o tipo de caixa a estudar, nomeadamente, a caixa de potencial infinito e a caixa com uma das paredes finita.

- Programa POCOINF: Permite estudar a caixa unidimensional de paredes infinitas. Apresenta inicialmente duas páginas de texto introdutório e uma lista de opções que permite seleccionar os parâmetros, cuja influência nas funções e energias se pretende observar.

- Programa CAIXA N: Mostra a influência do nº quântico nas funções e energias da partícula, para um dado comprimento da caixa. Apresenta lado a lado o gráfico de duas funções de estado e respectivas funções densidade de probabilidade, com os $\mathrm{n}^{\text {os }}$ quânticos escolhidos pelo utilizador $(\mathrm{N}$ máximo $=9$ ). Apresenta ainda para cada caso, o valor da energia em unidades de $\mathrm{h}^{2} / 8 \mathrm{~mL}^{2}$.

- Programa CAIXA L: Mostra a influência do comprimento da caixa nas funções e energias da partícula. Exibe o gráfico da função com o $\mathrm{n}^{\circ}$ quântico escolhido, assim como a respectiva energia, permitindo ao utilizador variar o comprimento da caixa de um modo contínuo, desde $-50 \%$ até $+100 \%$. Apresenta em seguida a nova energia e a respectiva função de estado sobreposta à inicial, sendo possível comparar as energias para os dois sistemas, através de dois gráficos apresentados lado a lado.

- Programa POCOFIN: Trata o problema da caixa com uma das paredes de potencial constante $\mathrm{U}$ (Estados ligados, $\mathrm{U}>$ E). Apresenta inicialmente duas páginas de texto introdutório e uma lista de opções onde se pode seleccionar como parâmetro a variar, o $\mathrm{n}^{\circ}$ quântico $\mathrm{N}$ ou o potencial da parede U. O parâmetro U permite uma variação discreta com os valores $1.0,1.5$ e $2.0 \mathrm{eV}$, e o $\mathrm{n}^{\mathrm{o}}$ quântico $\mathrm{N}$ tem o valor máximo de 4. As funções são rapidamente apresentadas, assim como as suas características (valores da energia E, de $\mathrm{N}$ e de $\mathrm{U}$ ), sendo possível comparar os níveis energéticos de dois sistemas, através de dois gráficos apresentados lado a lado.

\section{Bibliografia}

- J. P. Lowe, Quantum Chemistry, Academic Press, London, 1978.

- R. Dony, Graphisme Scientifique sur Micro-Ordinateur, Masson, Paris, 1986.

- S. J. Formosinho, A. J. C. Varandas, Estrutura e Reactividade Molecular, Fundação Calouste Gulbenkian, Lisboa, 1986.

- P. W. Atkins, Molecular Quantum Mechanics, Oxford University Press, Oxford, 1983. 


\section{Convite à Reflexão...}

\section{Solução Homogénea}

Correm as ondas apressadas

montanhas de moléculas

cada uma estupidamente metida na sua vida

a trilióes de distância

formando em uníssono a branca espuma.

\section{Eras sobre eras}

antes de quaisquer olhos as poderem ver

ano após ano

batendo atroadoramente na praia, como agora.

Para quem, para quê?

Num planeta morto

sem nenhuma vida para alimentar.

Incessantemente

torturadas pela energia

prodigiosamente esbanjadas pelo sol

atiradas para o espaço.

Uma migalha põe o mar a bramir.

Lá bem no fundo do mar

todas as partículas repetem

os padrões umas das outras

até novas e mais complexas se formarem.

Originam outras iguais a si

e um novo bailado começa.

Crescendo em tamanho e complexidade

coisas vivas

massas de átomos

$A D N$, proteínas

bailando num esquema cada vez mais intrincado.

Saídos do berço

para terra seca

eis aqui

erguendo-se:

átomos com consciência;

matéria com curiosidade.

Ali junto ao mar,

maravilhando-se com a maravilha: eu

um universo de átomos

um átomo no universo. 